\title{
The impact of nodal status in major salivary gland carcinoma: A multicenter experience and proposal of a novel N-classification
}

\author{
Davide Lombardi $^{\text {a,*,1, Michele Tomasoni }}{ }^{a}$, Alberto Paderno ${ }^{a}$, Davide Mattavelli ${ }^{a}$, \\ Marco Ferrari $^{\mathrm{a}, \mathrm{b}}$, Simonetta Battocchio ${ }^{\mathrm{c}}$, Francesco Missale ${ }^{\mathrm{d}, \mathrm{e}}$, Francesco Mazzola ${ }^{\mathrm{d}}$, \\ Giorgio Peretti ${ }^{\mathrm{d}, \mathrm{f}}$, Davide Mocellin ${ }^{\mathrm{d}}$, Daniele Borsetto ${ }^{\mathrm{g}}$, Jonathan M. Fussey ${ }^{\mathrm{g}}$, Paul Nankivell ${ }^{\mathrm{g}}$, \\ Nikoleta Skalidi ${ }^{\mathrm{g}}$, Mario Bussi ${ }^{\mathrm{h}}$, Leone Giordano ${ }^{\mathrm{h}}$, Andrea Galli ${ }^{\mathrm{h}}$, Gianluigi Arrigoni ${ }^{\mathrm{i}}$, \\ Elena Raffetti ${ }^{j}$, Paul Pracy ${ }^{g}$, Vincent Vander Poorten ${ }^{\mathrm{k}, 1}$, Piero Nicolai ${ }^{\mathrm{b}, 1}$

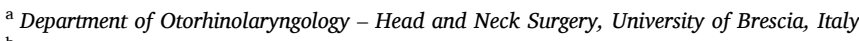 \\ ${ }^{\mathrm{b}}$ Section of Otorhinolaryngology - Head and Neck Surgery, Department of Neurosciences, University of Padua, Padua, Italy \\ ${ }^{\mathrm{c}}$ Unit of Pathology, University of Brescia, Italy \\ ${ }^{\mathrm{d}}$ Unit of Otorhinolaryngology - Head and Neck Surgery, IRCCS Ospedale Policlinico San Martino, Genoa, Italy \\ ${ }^{\mathrm{e}}$ Department of Molecular and Translational Medicine, University of Brescia, Brescia, Italy \\ ${ }^{\mathrm{f}}$ Department of Surgical Sciences and Integrated Diagnostics (DISC), University of Genoa, Genoa, Italy \\ ${ }^{g}$ Department of ENT/Head and Neck Surgery, Queen Elizabeth University Hospital Birmingham, Birmingham, United Kingdom \\ ${ }^{\mathrm{h}}$ Department of Otorhinolaryngology, IRCCS San Raffaele Scientific Institute, Milan, Italy \\ ${ }^{\mathrm{i}}$ Unit of Pathology, IRCCS San Raffaele Scientific Institute, Milan, Italy \\ ${ }^{\mathrm{j}}$ Epidemiology and Public Health Intervention Research Group (EPHIR), Department of Global Public Health, Karolinska Institutet, Stockholm, Sweden \\ ${ }^{\mathrm{k}}$ Otorhinolaryngology - Head and Neck Surgery and Department of Oncology, Section Head and Neck Oncology, University Hospitals Leuven, KU Leuven, Leuven, \\ Belgium
}

\section{A R T I C L E I N F O}

\section{Keywords:}

Major salivary gland cancer

Nodal metastasis

Parotid gland

Submandibular gland

Tumor staging

Parotid lymph-nodes

Positive lymph-node number

Positive lymph-node diameter

Extra-nodal extension

Head and neck cancer

\begin{abstract}
A B S T R A C T
Objectives: Despite differences in oncological behavior, the 8th edition of AJCC TNM staging currently proposes the same N-classification for major salivary glands (MSG) carcinoma and squamous cell carcinoma of the upper aerodigestive tract. The present study aims to investigate a more reliable definition of N-categories for MSG carcinoma.

Materials and methods: A retrospective multicenter study was performed, including 307 patients treated for primary MSG carcinoma from 1995 to 2019. Outcome measures included overall survival (OS), disease specific survival, and local, regional, and distant recurrence. Survival analysis was performed using log-rank test and Cox proportional-hazards model. Overall number $(\mathrm{ON})$ and largest diameter (LD) of nodal metastases, including intra-parotid metastases, were considered to develop three novel proposals of N-classification; their performance were compared with the current TNM staging using Akaike information criterion (AIC), Bayesian information criterion (BIC), and Nagelkerke pseudo- $\mathrm{R}^{2}$.

Results: Intra-parotid nodes, ON and LD of nodal metastases emerged as major prognosticators for OS, while extra-nodal extension did not impact on any survival. The current N-classification did not show a satisfactory OS stratification. Three novel $\mathrm{N}$-classifications were developed according to number of metastatic nodes ( 0 vs $1-3$ vs $\geq 4$ ) and/or their maximum diameter ( $<20 \mathrm{~mm}$ vs $\geq 20 \mathrm{~mm}$ ). They all showed better accuracy in OS stratification, and achieved better AIC, BIC and Nagelkerke pseudo- $\mathrm{R}^{2}$ indices when compared to current $\mathrm{N}$ classification.

Conclusion: All the proposed N-classifications improved OS stratification and could help in defining a specific Nclassification for MSG carcinoma. Their validation and assessment in an external cohort is needed.
\end{abstract}

\footnotetext{
* Corresponding author at: Department of Otorhinolaryngology, Head and Neck Surgery, University of Brescia, Piazzale Spedali Civili 1, 25100 Brescia, Italy. E-mail address: davinter@libero.it (D. Lombardi).

1 MSGS, Multidisciplinary Salivary Gland Society, Geneva, Switzerland.
} 


\section{Introduction}

Malignant major salivary gland (MSG) tumors are extremely rare, only accounting for a small percentage of all head and neck cancers. Nodal involvement is considered as one of the most important factors affecting survival in MSG carcinoma [1-7].

The current AJCC 8th TNM staging system proposes the same Ncategories be used for both MSG and upper aerodigestive tract squamous cell carcinoma (SCC) [8]. This is despite significant differences in biological behavior, treatment modalities and outcomes between the two entities. Consequently, there are several limitations to the MSG N-classification. Firstly, the TNM classification does not consider the involvement of parotid lymph-nodes, despite them being the only involved station in a non-negligible proportion of patients [1,9-11]. In addition, parotid nodal metastasis per se may increase the risk of recurrence $[9,10]$. Secondly, contralateral nodal metastasis in MSG carcinoma is considered so rare as to be anecdotal [2,12]. This supports the argument that only the ipsilateral neck should be treated $[2,13,14]$.

Finally, the prognostic relevance of nodal dimensions and extranodal extension (ENE) are probably the most important nodal predictors in upper aerodigestive tract SCC, yet their significance in MSG carcinoma is less clear [9,15-18]. In MSG carcinoma, the nodal disease burden, defined by nodal ratio (NR) and/or number of lymph-nodes involved, may have a more significant prognostic relevance [14-18].

The aim of this study is to present a multicenter experience in the treatment of primary MSG carcinoma, with a specific focus on the prognostic significance of different nodal factors. A novel N-classification specific for primary MSG carcinoma will also be proposed.

\section{Materials and methods}

A multicenter retrospective analysis of patients affected by carcinoma of the MSG was conducted at the Departments of OtolaryngologyHead and Neck Surgery of the University of Brescia, Brescia, Italy; Queen Elizabeth Hospital, Birmingham, England; University Vita-Salute San Raffaele, Milan, Italy; and University of Genova, Genoa, Italy. Patients affected by MSG primary epithelial malignant tumor receiving surgery with a curative intent as upfront treatment from January 1995 to September 2019 were considered eligible. Exclusion criteria were previous treatment and metastatic tumor to the MSG. Data concerning survival and recurrence outcomes were retrieved from mortality registries, outpatient visits, and radiological follow-up. Data management and study accomplishment are in accordance with principles stated in the Declaration of Helsinki; the study was approved by the local ethics committee (NP-2066, WV-H\&N Cancer).

\section{Demographics, clinical and pathological data}

Data concerning demographics (gender, age), clinical presentation (facial palsy, skin ulceration, pain), imaging, surgical treatment, pathology, and adjuvant therapy were systematically collected. Histopathological features retrieved included histologic type and grading, margin status, local extension, perineural (PNI) and lympho-vascular invasion (LVI), and characteristics of intraparotid and cervical metastatic nodes (largest diameter, number, ENE). Grading of MucoEpidermoid Carcinoma (MEC) and Adenoid-Cystic Carcinoma (AdCC) was assessed according to the Brandwein and Perzin-Szanto classification, respectively [20-23]. Patients with pre-operative evidence of cervical nodal metastasis received a comprehensive (radical or modified radical) neck dissection (ND). Conversely, patients without preoperative evidence of cervical metastasis were treated with a selective or super-selective ND or were observed according to the specific risk profile for occult nodal involvement [24]. Lymph-nodes within the parotid gland were considered part of the cervical lymphatic system and classified as a specific nodal level; the overall count of Nodal Metastases (NM) included both intraparotid and cervical nodes. Tumor staging of all patients was re-evaluated according to the 8th edition of the TNM Classification of Malignant Tumors [8].

\section{Statistical analysis}

Characteristics of patients were expressed in terms of mean, standard deviation, median, range of values and percentages. Time to death, first recurrence (any site) and the latest available clinical or radiological evaluation (censored observations) were considered as outcomes. Overall (OS), Disease-Specific (DSS), Recurrence-Free (RFS), Local Recurrence-Free (LRFS), Regional Recurrence-Free (RRFS), and Distant Recurrence-Free (DRFS) Survivals were evaluated using Kaplan-Meier survival curves. Relation between characteristics of intraparotid and cervical NM, and with ENE, was established using logistic regression. Furthermore, the role of demographic, clinical, and pathologic characteristics on survival was evaluated using the Log-rank test (univariate analysis).

The continuous variables "overall number" (ON) and "largest diameter" (LD) of NM were categorized applying cutoff values determined using the X-tile software (3.6.1 - Yale University, New Haven, CT, USA), according to the minimum p-value and the maximum $\chi^{2}$ [25]. Univariate and multivariable Cox proportional hazard model was performed to analyze the prognostic role of ON and LD on OS. Results were expressed in terms of Hazard Ratio (HR) and 95\% confidence intervals (CI).

Three proposals for a new nodal classification of the TNM were developed, considering ON, LD or a combination of both. Risk stratification in terms of survival and recurrence of each nodal classification was assessed with the log-rank test and Kaplan Meier method. Correction for multiple comparisons according to Holm method was performed for each nodal classification. Akaike information criterion (AIC), Bayesian information criterion (BIC), and Nagelkerke pseudo- $\mathrm{R}^{2}$ index were calculated and compared between the new proposals of $\mathrm{N}$ categorization and the current $\mathrm{N}$ classification (TNM 8th edition).

Multivariable Cox proportional hazard model was adopted to determine the independent prognostic role of proposed $\mathrm{N}$-classifications in $\mathrm{pN}+$ patients. Schoenfeld residuals were evaluated for the assessment of proportional hazards assumption; variance inflation factors $(\mathrm{VIF}<5)$ were estimated to exclude multi-collinearity between covariates.

Statistical analysis was performed using R (version 3.6.3, R Foundation for Statistical Computing, Vienna, Austria); $\mathrm{p}$ values $<0.05$ were considered statistically significant.

\section{Results}

\section{Demographics and clinical presentation}

Three-hundred-seven patients were included in the study. The mean age was 58.9 years (SD 17.1, range 6-91), with an almost equal gender distribution (1.03:1 male to female ratio). Patients were affected by primary parotid and submandibular carcinoma in $93.2 \%$ and $6.8 \%$ cases, respectively. Preoperative evidence of cervical and intraparotid node involvement $(\mathrm{cN}+)$ was identified in $23.3 \%$ and $14.7 \%$ of cases, respectively. The cohort characteristics are summarized in Table 1.

\section{Histopathology of nodal metastasis}

The number and largest dimension of cervical and parotid lymphnodes are detailed in Table 1 . Nodal metastasis at final pathology report $(\mathrm{pN}+$ ) were observed in 95/303 (31.4\%) patients: overall, intraparotid and cervical NM were diagnosed in 66/298 (22.1\%) and $74 / 303(24.4 \%)$ cases, respectively. Preoperative imaging failed to identify occult cervical and intraparotid metastasis in $20.0 \%$ and $20.1 \%$ of cases, respectively. Almost half of $\mathrm{pN}+$ patients had both intraparotid and cervical node metastasis (45/95, 47.4\%), whereas exclusive intraparotid and cervical node metastasis were less frequently observed (21/ 
Table 1

Descriptive statistics of the cohort of patients. mRND - modified Radical Neck Dissection, RND - Radical Neck Dissection, SD - Standard Deviation, SND - Selective Neck Dissection.

\begin{tabular}{|c|c|c|c|}
\hline Variable & & N. & $\%$ \\
\hline \multirow[t]{4}{*}{ Participant Institutions } & Brescia & 203 & $66.1 \%$ \\
\hline & Birmingham & 46 & $15.0 \%$ \\
\hline & San Raffaele - Milan & 45 & $14.7 \%$ \\
\hline & Genova & 13 & $4.2 \%$ \\
\hline Mean age \pm SD (range) - yr & $58.9 \pm 17.1(6-91)$ & & \\
\hline \multirow[t]{2}{*}{ Gender } & Female & 151 & $49.2 \%$ \\
\hline & Male & 156 & $50.8 \%$ \\
\hline \multirow[t]{2}{*}{ Primary site } & Submandibular gland & 21 & $6.8 \%$ \\
\hline & Parotid gland & 286 & $93.2 \%$ \\
\hline $\begin{array}{l}\text { Mean } \mathrm{H} \text { time } \pm \text { SD (range) - } \\
\text { days }\end{array}$ & $5 \pm 4.2(1-34)$ & & \\
\hline \multirow[t]{5}{*}{ Clinical signs on presentation } & Pain & $55 /$ & $20.7 \%$ \\
\hline & & 265 & \\
\hline & Skin alteration & $17 /$ & $6.2 \%$ \\
\hline & & 273 & \\
\hline & Facial palsy & $\begin{array}{l}36 / \\
273\end{array}$ & $13.2 \%$ \\
\hline \multirow[t]{5}{*}{$\begin{array}{l}\text { Parotid tumor extension at } \\
\text { the time of surgery }\end{array}$} & Superficial lobe & $\begin{array}{l}154 / \\
266\end{array}$ & $57.9 \%$ \\
\hline & Deep lobe & $\begin{array}{l}35 / \\
266\end{array}$ & $13.2 \%$ \\
\hline & Extensive/both lobes & $\begin{array}{l}49 / \\
266\end{array}$ & $18.4 \%$ \\
\hline & Masseteric process & $\begin{array}{l}12 / \\
266\end{array}$ & $4.5 \%$ \\
\hline & Parotid tail & $\begin{array}{l}16 / \\
266\end{array}$ & $6.0 \%$ \\
\hline \multirow[t]{7}{*}{ Parotid surgery } & $\begin{array}{l}\text { Less than superficial } \\
\text { parotidectomy }\end{array}$ & $\begin{array}{l}3 / \\
274\end{array}$ & $1.1 \%$ \\
\hline & Superficial parotidectomy & $\begin{array}{l}121 / \\
274\end{array}$ & $44.1 \%$ \\
\hline & $\begin{array}{l}\text {-Extended superficial } \\
\text { parotidectomy }\end{array}$ & (12) & $(9.9 \%)$ \\
\hline & Total parotidectomy & $\begin{array}{l}101 / \\
274\end{array}$ & $36.9 \%$ \\
\hline & $\begin{array}{l}\text {-Extended total } \\
\text { parotidectomy }\end{array}$ & (24) & $(23.8 \%)$ \\
\hline & Radical parotidectomy & $\begin{array}{l}49 / \\
274\end{array}$ & $17.9 \%$ \\
\hline & $\begin{array}{l}\text {-Extended radical } \\
\text { parotidectomy }\end{array}$ & (33) & $(67.3 \%)$ \\
\hline \multirow[t]{6}{*}{$\begin{array}{l}\text { Nodal dissection for parotid } \\
\text { malignancies }\end{array}$} & No ND performed & $\begin{array}{l}126 / \\
282\end{array}$ & $44.7 \%$ \\
\hline & ND performed & $\begin{array}{l}156 / \\
282\end{array}$ & $55.3 \%$ \\
\hline & $\begin{array}{l}\text {-Super-selective ND (II or } \\
\text { II-III) }\end{array}$ & $(64)$ & $(41.0 \%)$ \\
\hline & -Selective ND (II-IV) & (48) & $(30.8 \%)$ \\
\hline & $-\mathrm{mRND} / \mathrm{RND}(\mathrm{I}-\mathrm{V})$ & (42) & $(26.9 \%)$ \\
\hline & -Not otherwise specified & (2) & $(1.3 \%)$ \\
\hline \multirow{5}{*}{$\begin{array}{l}\text { Nodal dissection for } \\
\text { submandibular } \\
\text { malignancies }\end{array}$} & No ND performed & $5 / 21$ & $23.8 \%$ \\
\hline & ND performed & $\begin{array}{l}16 / \\
21\end{array}$ & $76.2 \%$ \\
\hline & -Super-selective ND (I) & (7) & $(43.7 \%)$ \\
\hline & -Selective ND (I-III/IV) & (4) & $(25.0 \%)$ \\
\hline & $-\mathrm{mRND} / \mathrm{RND}(\mathrm{I}-\mathrm{V})$ & (5) & $(31.3 \%)$ \\
\hline \multirow[t]{6}{*}{ Histology } & $\begin{array}{l}\text { Mucoepidermoid Carcinoma } \\
\text { (MEC) }\end{array}$ & $\begin{array}{l}63 / \\
307\end{array}$ & $20.5 \%$ \\
\hline & Acinic Cell Carcinoma & $48 /$ & $15.6 \%$ \\
\hline & (AcCC) & 307 & \\
\hline & $\begin{array}{l}\text { Salivary Duct Carcinoma } \\
\text { (SDC) }\end{array}$ & $\begin{array}{l}37 / \\
307\end{array}$ & $12.0 \%$ \\
\hline & $\begin{array}{l}\text { Adenoid Cystic Carcinoma } \\
\text { (AdCC) }\end{array}$ & $\begin{array}{l}35 / \\
307\end{array}$ & $11.4 \%$ \\
\hline & & & $8.1 \%$ \\
\hline
\end{tabular}

Table 1 (continued)

\begin{tabular}{|c|c|c|c|}
\hline \multicolumn{2}{|l|}{ Variable } & \multirow{2}{*}{$\frac{N .}{25 /}$} & \multirow[t]{2}{*}{$\%$} \\
\hline & Adenocarcinoma Not & & \\
\hline & Otherwise Specified (ADC & 307 & \\
\hline & NOS) & & \\
\hline & Carcinoma Ex-Pleomorphic & $31 /$ & $10.1 \%$ \\
\hline & Adenoma (CEPA) & 307 & \\
\hline & Basal cell adenocarcinoma & $10 /$ & $3.2 \%$ \\
\hline & & 307 & \\
\hline & Poorly differentiated & $10 /$ & $3.2 \%$ \\
\hline & carcinoma & 307 & \\
\hline & Myoepithelial carcinoma & $\begin{array}{l}10 / \\
307\end{array}$ & $3.2 \%$ \\
\hline & Epithelial-myoepithelial & 9/ & $2.9 \%$ \\
\hline & carcinoma & 307 & \\
\hline & Primitive salivary squamous & 8/ & $2.6 \%$ \\
\hline & cell carcinoma & 307 & \\
\hline & Mammary analogue & 5/ & $1.6 \%$ \\
\hline & secretory carcinoma & 307 & \\
\hline & Oncocytic carcinoma & 5/ & $1.6 \%$ \\
\hline & & 307 & \\
\hline & Carcinosarcoma & 4/ & $1.3 \%$ \\
\hline & & 307 & \\
\hline & Lymphoepitelial carcinoma & $3 /$ & $1.0 \%$ \\
\hline & & 307 & \\
\hline & Intraductal carcinoma & 2/ & $0.6 \%$ \\
\hline & & 307 & \\
\hline & Sebaceous adenocarcinoma & $1 /$ & $0.3 \%$ \\
\hline & & 307 & \\
\hline & Polymorphous & 1/ & $0.3 \%$ \\
\hline & adenocarcinoma & 307 & \\
\hline \multirow[t]{6}{*}{ Grading } & Low-grade & $138 /$ & $46.5 \%$ \\
\hline & & 297 & \\
\hline & Intermediate-grade & $39 /$ & $13.1 \%$ \\
\hline & & 297 & \\
\hline & High-grade & $120 /$ & $40.4 \%$ \\
\hline & & 297 & \\
\hline $\begin{array}{l}\text { Mean primary tumor major } \\
\text { diameter } \pm \text { SD (range) - } \\
\text { mm }\end{array}$ & $27.3 \pm 17.6(3-100)$ & & \\
\hline \multirow[t]{4}{*}{ Margin status } & R0 & $179 /$ & $58.7 \%$ \\
\hline & & 305 & \\
\hline & $\mathrm{R} 1$ & $126 /$ & $41.3 \%$ \\
\hline & & 305 & \\
\hline \multirow[t]{11}{*}{ Extraglandular Extension } & Absent & $187 /$ & $64.3 \%$ \\
\hline & & 291 & \\
\hline & Present & $104 /$ & $35.7 \%$ \\
\hline & & 291 & \\
\hline & -Facial Nerve infiltration & (35) & $12.4 \%$ \\
\hline & -Connective tissue/fat & $(56)$ & $20.7 \%$ \\
\hline & infiltration & & \\
\hline & -Muscles infiltration & (28) & $10.4 \%$ \\
\hline & -Bone infiltration & (8) & $2.9 \%$ \\
\hline & -Cartilage infiltration & (4) & $1.5 \%$ \\
\hline & -Dermal/epidermal & (34) & $11.4 \%$ \\
\hline \multirow{4}{*}{ Perineural invasion } & Pno & $173 /$ & $57.3 \%$ \\
\hline & & 302 & \\
\hline & Pn1 & $129 /$ & $42.7 \%$ \\
\hline & & 302 & \\
\hline \multirow[t]{4}{*}{ Lymphovascular invasion } & V0 & 194/ & $69.0 \%$ \\
\hline & & 281 & \\
\hline & LV1VI+ & $87 /$ & $31.0 \%$ \\
\hline & & 281 & \\
\hline \multirow[t]{8}{*}{ Nodal metastasis } & No nodal metastasis & 208/ & $68.6 \%$ \\
\hline & & 303 & \\
\hline & Overall pN+ (cervical and/or & 95/ & $31.4 \%$ \\
\hline & intraparotid) & 303 & \\
\hline & -Cervical pN+ & $74 /$ & $24.4 \%$ \\
\hline & & 303 & \\
\hline & -Intraparotid $\mathrm{pN}+$ & $66 /$ & $22.1 \%$ \\
\hline & & 298 & \\
\hline Location of nodal metastasis & Exclusive intraparotid pN+ & & $22.1 \%$ \\
\hline
\end{tabular}

(continued on next page) 
Table 1 (continued)

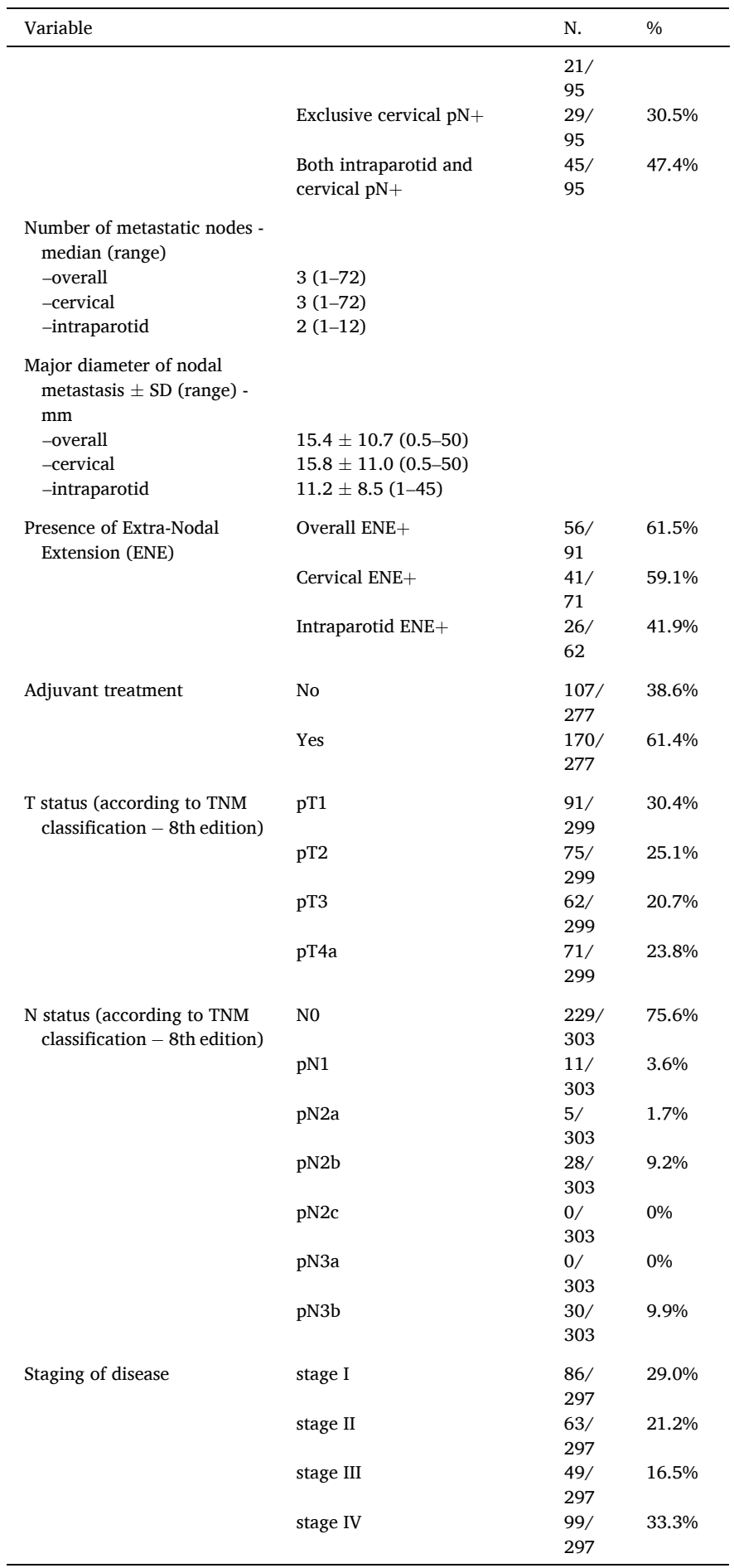

95 [22.1\%] and 29/95 [30.5\%], respectively). Overall, the median number of metastatic nodes was 4 (range: $1-72$; mean: 9.95), with a median largest diameter of $13.5 \mathrm{~mm}$ (range: $0.5-50 \mathrm{~mm}$; mean: 15.4 $\mathrm{mm})$. ENE was observed in 41/71 (59.2\%) and 26/62 (41.9\%) of cervical and intraparotid NM, respectively. The number of intraparotid NM was strongly associated with the presence of cervical NM at logistic regression ( $\mathrm{p}<0.001$; Fig.S1). Frequency of cervical NM involvement was $100 \%$ in patients bearing 4 or more intraparotid NM. The ON of metastatic nodes and their LD were associated with a higher rate of ENE at logistic regression ( $\mathrm{p}=0.04$ and $\mathrm{p}=0.05$, respectively; Fig. S2, S3).

\section{Staging}

The $\mathrm{pT}$ and $\mathrm{pN}$ categories, as well as the definitive staging of disease, are shown in Table 1 . Cervical NM were diagnosed in $24.4 \%$ of patients. The majority of $\mathrm{pN}+$ cases were classified as $\mathrm{pN} 2 \mathrm{~b}(9.2 \%)$ or $\mathrm{pN} 3 \mathrm{~b}$ (9.9\%); pN1 and $\mathrm{pN} 2 \mathrm{a}$ were rarely observed $(3.6 \%$ and $1.7 \%$, respectively). No bilateral NM (pN2c category) nor NM with the largest diameter $>60 \mathrm{~mm}$ (pN3a category) were observed.

\section{Survival analysis}

Follow-up data were available for 295 patients (12 lost at follow-up, 3.9\%). Median follow-up was 51 months (inter-quartile range [IQR]: 82.7, mean: 75.8 months, range: 3-292). At the end of the present study (April 2020), 63.4\% of patients were alive without disease and $6.4 \%$ were alive with recurrence. Patients died more frequently because of the disease $(18.0 \%)$ rather than other causes $(9.8 \%)$. In $2.4 \%$ of cases, cause of death was not established. Disease persistence or recurrence was found in $27.0 \%$ of patients, with a median interval to first recurrence of 16.5 months (range: 1-126 months). Local failure was diagnosed in $12.1 \%$ (median interval: 14 months, range: 1-59 months), regional failure in 7.4\% (median interval: 12 months, range: $1-56$ months), and distant metastasis in $21.6 \%$ of cases (median interval: 16 months, range: 0-202 months). Survival data and univariate analysis of major prognostic factors on survival and recurrence are shown in Table S1 and S2. Of note, tumor grading, PNI, LVI, margin status and pT categories (pT14) were associated with a worse outcome in terms of OS, DSS and RFS.

Current pN classification (TNM 8th edition) was not able to correctly stratify patients: at multiple pairwise comparison only the difference between survival of $\mathrm{pNO}$ vs pN2b and pN0 vs pN3b patients were statistically significant (adjusted p-value using the Holm method). In pN+ patients no statistically significant difference could be appreciated according to current classification (Fig. S4).

ENE did not significantly impact on OS ( $p=0.194$, Fig. 1), DSS ( $\mathrm{p}=$ $0.134), R F S(p=0.159)$, LRFS $(p=0.229)$, RRFS $(p=0.941)$ and DRFS

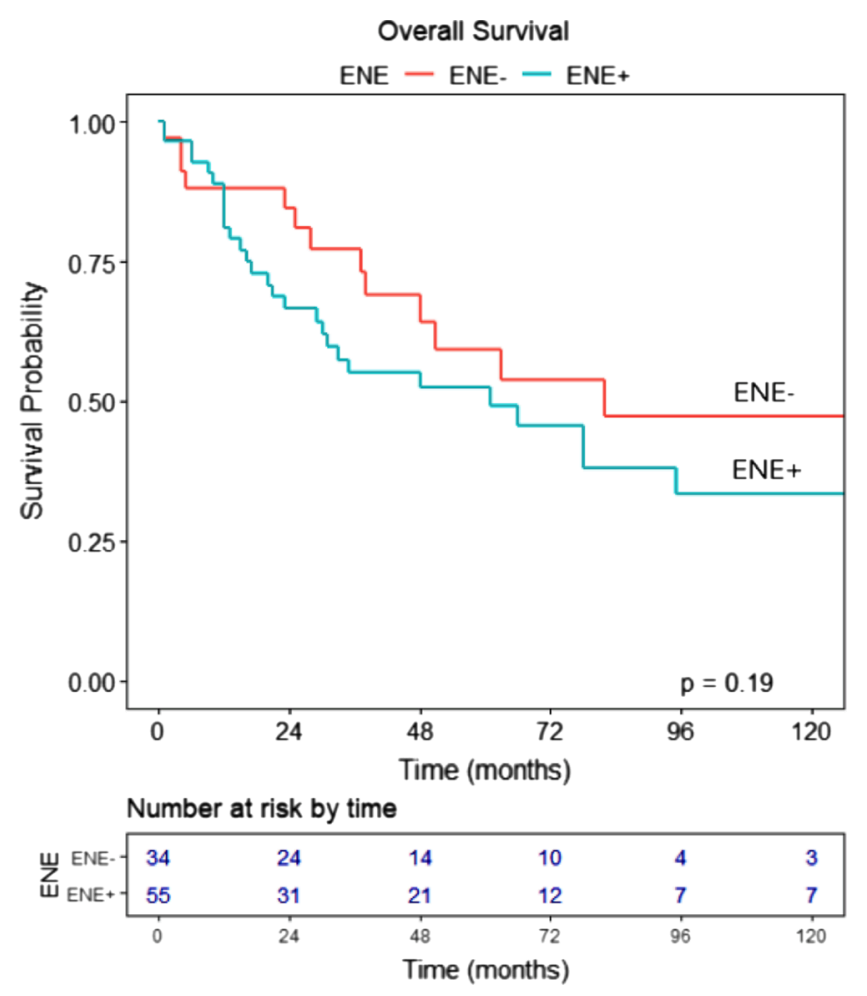

Fig. 1. Kaplan Meier survival curves showing no statistically significant difference between $\mathrm{pN}+\mathrm{ENE}+$ and $\mathrm{pN}+$ ENE- patients. 
( $\mathrm{p}=0.265$ ). Metastasis to cervical nodes was a strong negative prognostic factor for all survival outcomes $(p<0.001)$. The presence of intraparotid NM was also associated with a worse outcome in all survival estimates ( $\mathrm{p} \leq 0.01$ ). Univariate analysis using Cox proportional regression model showed that ON (HR: $1.04,95 \%$ CI 1.02-1.05; p < 0.001 ) and LD (HR: 1.73 per cm increase, 95\% CI 1.48-2.02; $\mathrm{p}<0.001$ ) of nodal metastasis significantly affected OS.

Multivariable analyses (Table 2) proved the independent prognostic role of $\mathrm{ON}$ and LD, adjusted for major prognostic covariates, in terms of OS; conversely, they confirmed the negligible role of ENE and the inadequacy of current $\mathrm{N}$ classification (TNM 8th ed.).

\section{Proposal of novel $N$ classifications}

Considering the limited value of different $\mathrm{pN}+$ categories in survival stratification, data on NM were thoroughly analyzed to build a more reliable classification of $\mathrm{N}$ status. Intraparotid nodes were considered within the overall count of dissected nodes, laterality was not considered since no cases of contralateral NM were encountered. Similarly, ENE was not considered as a prognostic factor since it was not a statistically significant variable at univariate and multivariable analyses. The ON and LD of metastatic lymph-nodes were therefore the only factors considered.

With the X-tile analysis (Fig. 2), the optimal cut-offs in terms of OS for ON and LD of nodal metastasis were determined. According to minimum $\mathrm{p}$ - and maximum $\chi^{2}$ - values of log rank test, ideal cutoff point in the ON was $\geq 4\left(\mathrm{p}=0.001, \chi^{2}=10.17\right)$, and in the LD was $\geq 20 \mathrm{~mm}$ $\left(\mathrm{p}<0.001, \chi^{2}=11.53\right)$.

According to these findings, nodal classifications based on the ON and LD of NM were formulated.

\section{$\mathrm{N}$-classification 1 (based on overall number of metastatic nodes)}

- N0: no intraparotid or cervical nodal metastasis,

- N1: $<4$ intraparotid and/or cervical nodal metastasis,

- N2: $\geq 4$ intraparotid and/or cervical nodal metastasis.
$\mathrm{N}$-classification 2 (based on largest diameter of the overall count of metastatic nodes)

- N0: no intraparotid or cervical nodal metastasis,

- N1: nodal metastasis with the largest diameter $<20 \mathrm{~mm}$,

- N2: nodal metastasis with the largest diameter $\geq 20 \mathrm{~mm}$.

Combining data of the ON and LD of metastatic nodes, 4 classes of $\mathrm{pN}+$ patients could be identified:

- patients with 1-3 nodal metastasis with the largest diameter $<20$ $\mathrm{mm}$,

- patients with 1-3 nodal metastasis with the largest diameter $\geq 20$ $\mathrm{mm}$,

- patients with $\geq 4$ nodal metastasis with the largest diameter $<20$ $\mathrm{mm}$,

- patients with $\geq 4$ nodal metastasis with the largest diameter $\geq 20$ $\mathrm{mm}$.

According to pattern of survival at univariate analysis (Fig. S5), these classes were reformulated in terms of nodal disease burden, conceiving a third classification, as follows.

$\mathrm{N}$-classification 3 (based on number and largest diameter of the overall count of nodal metastasis)

- N0: no intraparotid or cervical nodal metastasis,

- N1 (low nodal disease burden): 1-3 nodal metastasis with the largest diameter $<20 \mathrm{~mm}$,

- N2 (high nodal disease burden): $\geq 4$ nodal metastasis and/or at least one nodal metastasis with the largest diameter $\geq 20 \mathrm{~mm}$.

All indices estimating the trade-off between prediction accuracy and simplicity of the model (i.e., AIC and BIC) and those expressing how comprehensively models can explain data (i.e., Nagelkerke pseudo-R) were in favor of the novel $\mathrm{N}$-classifications as compared to the 8 th

Table 2

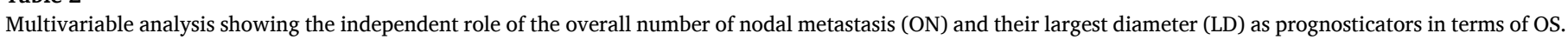

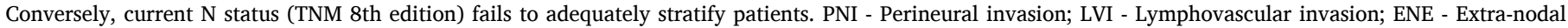
extension.

\begin{tabular}{|c|c|c|c|c|c|c|c|c|c|c|}
\hline \multirow[t]{3}{*}{ Variable } & & \multicolumn{9}{|l|}{ OS } \\
\hline & & \multicolumn{3}{|c|}{ Multivariable analysis } & \multicolumn{3}{|c|}{ Multivariable analysis } & \multicolumn{3}{|c|}{ Multivariable analysis } \\
\hline & & HR & $95 \% \mathrm{CI}$ & p-value & HR & $95 \%$ CI & p-value & HR & $95 \% \mathrm{CI}$ & $\mathrm{p}$-value \\
\hline Age (years) & & 1.06 & $1.03-1.08$ & $<0.001$ & 1.06 & $1.04-1.08$ & $<0.001$ & 1.06 & $1.03-1.08$ & $<0.001$ \\
\hline \multirow{3}{*}{ Grading } & low & & & & & & & & & \\
\hline & intermediate & 1.75 & $0.62-4.90$ & 0.289 & 1.48 & $0.49-4.49$ & 0.49 & 1.74 & $0.63-4.84$ & 0.290 \\
\hline & high & 2.81 & $1.38-5.71$ & 0.004 & 2.87 & $1.32-6.25$ & 0.008 & 2.99 & $1.47-6.05$ & 0.002 \\
\hline \multirow[t]{4}{*}{ pT } & pT1 & & & & & & & & & \\
\hline & pT2 & 1.31 & $0.50-3.42$ & 0.584 & 1.22 & $0.44-3.35$ & 0.698 & 1.24 & $0.47-3.23$ & 0.664 \\
\hline & pT3 & 1.97 & $0.82-4.75$ & 0.132 & 2.26 & $0.94-5.43$ & 0.068 & 2.02 & $0.84-4.85$ & 0.116 \\
\hline & pT4 & 2.26 & $0.92-5.55$ & 0.075 & 2.35 & $0.94-5.88$ & 0.068 & 2.00 & $0.80-4.98$ & 0.137 \\
\hline \multirow[t]{2}{*}{ Surgical margins } & R0 & & & & & & & & & \\
\hline & $\mathrm{R} 1$ & 1.26 & $0.74-2.13$ & 0.393 & 1.22 & $0.70-2.12$ & 0.486 & 1.30 & $0.75-2.24$ & 0.349 \\
\hline \multirow[t]{2}{*}{ PNI } & Pno & & & & & & & & & \\
\hline & Pn1 & 1.31 & $0.61-2.82$ & 0.482 & 1.53 & $0.69-3.40$ & 0.293 & 1.26 & $0.62-2.56$ & 0.522 \\
\hline \multirow[t]{2}{*}{ LVI } & V0 & & & & & & & & & \\
\hline & V1 & 1.32 & $0.71-2.43$ & 0.376 & 0.96 & $0.48-1.90$ & 0.903 & 1.16 & $0.63-2.16$ & 0.630 \\
\hline \multirow[t]{3}{*}{ ENE } & N0 & & & & & & & & & \\
\hline & ENE- & 1.15 & $0.54-2.44$ & 0.708 & 0.75 & $0.30-1.87$ & 0.539 & - & - & - \\
\hline & ENE+ & 0.98 & $0.44-2.16$ & 0.951 & 0.74 & $0.26-2.06$ & 0.561 & - & - & - \\
\hline \multirow{2}{*}{\multicolumn{2}{|c|}{$\begin{array}{l}\text { Overall Number of nodal metastasis (ON) - N. } \\
\text { Largest Diameter of nodal metastasis (LD) }-\mathrm{cm}\end{array}$}} & 1.02 & $1.01-1.05$ & 0.045 & - & - & - & - & - & - \\
\hline & & - & - & - & 1.50 & $1.06-2.13$ & 0.023 & - & - & - \\
\hline \multirow[t]{5}{*}{ N staging (TNM 8th ed.) } & No & - & - & - & - & - & - & & & \\
\hline & N1 & - & - & - & - & - & - & 1.89 & $0.63-5.65$ & 0.254 \\
\hline & $\mathrm{N} 2 \mathrm{a}$ & - & - & - & - & - & - & 1.19 & $0.34-4.15$ & 0.781 \\
\hline & $\mathrm{N} 2 \mathrm{~b}$ & - & - & - & - & - & - & 1.53 & $0.71-3.29$ & 0.281 \\
\hline & $\mathrm{N} 3 \mathrm{~b}$ & - & - & - & - & - & - & 2.05 & $0.93-4.49$ & 0.074 \\
\hline
\end{tabular}



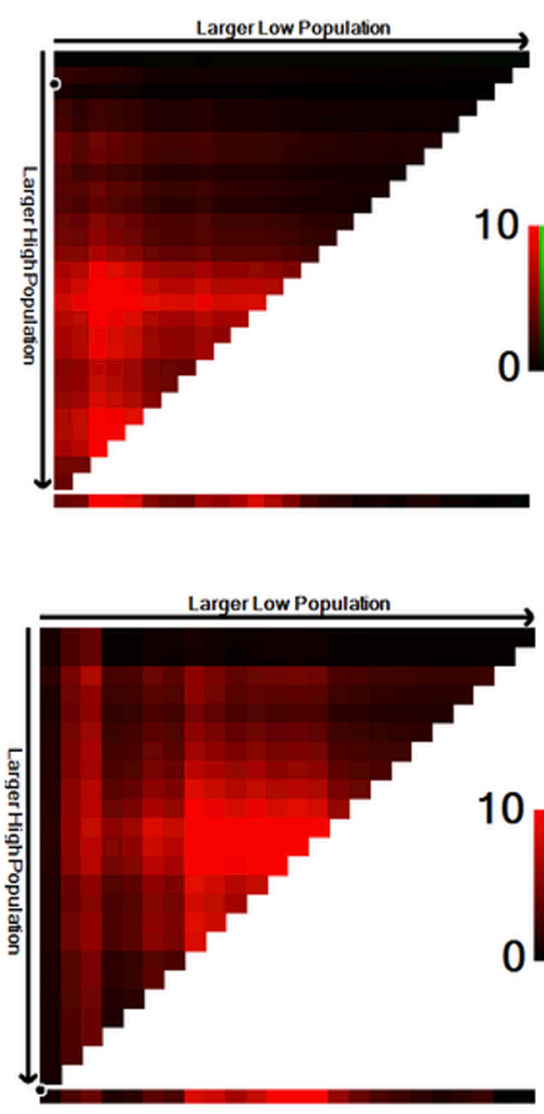
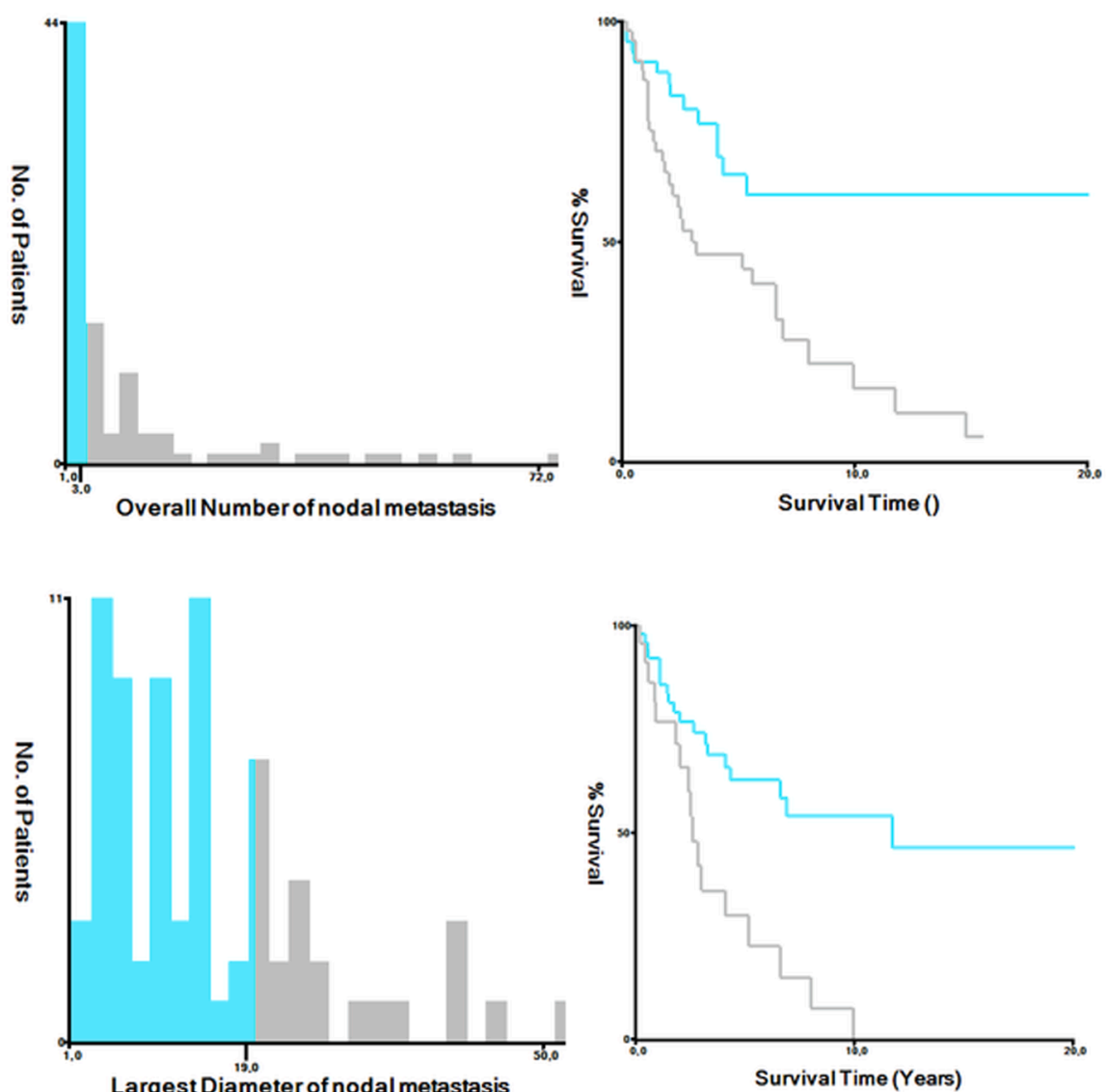

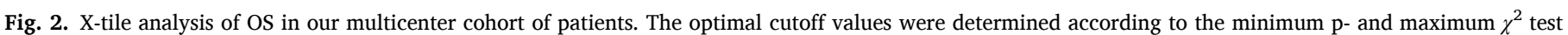
values: $\geq 4$ for overall number $\left(\mathrm{p}=0.001, \chi^{2}=10.17\right.$ ), and $\geq 20 \mathrm{~mm}$ for largest diameter of nodal metastasis $\left(\mathrm{p}<0.001, \chi^{2}=11.53\right.$ ).

TNM Edition N-classification (Table 3).

The univariate analysis (Fig. 3a-c, Table 3) showed that each of the 3 new nodal classifications effectively stratify patients in terms of survival outcomes ( $\mathrm{p}<0.001$ ). For each new proposal of $\mathrm{N}$ classification, a statistically significant difference between all sub-categories was demonstrated at multiple pairwise comparison (p-value adjusted for multiple comparisons using the Holm method). Further, to assess performance in survival stratification in $\mathrm{pN}+$ patients, nodal high-risk profiles ( $\mathrm{ON} \geq 4, \mathrm{LD} \geq 20 \mathrm{~mm}$, or high burden of nodal disease) adjusted for major prognostic covariates in the clinical setting,

Table 3

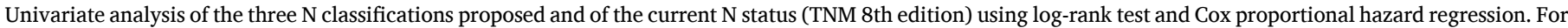

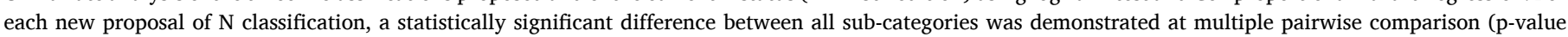

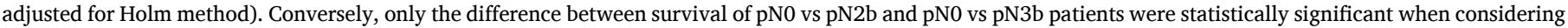
current N status (TNM 8th edition). * Nagelkerke pseudo- ${ }^{2}$.

\begin{tabular}{|c|c|c|c|c|c|c|c|c|c|}
\hline & \multicolumn{3}{|c|}{ Log-rank test } & \multicolumn{6}{|c|}{ Cox proportional hazard regression } \\
\hline & 5 -y OS & $95 \% \mathrm{CI}$ & p-value & HR & $95 \% \mathrm{CI}$ & p-value & AIC & BIC & pseudo- $\mathrm{R}^{2 *}$ \\
\hline \multicolumn{10}{|l|}{$\mathrm{N}$ classification 1} \\
\hline pNO & $83.1 \%$ & $77.6-89.1 \%$ & $<0.001$ & & & & 849.64 & 854.59 & 0.281 \\
\hline Overall number of $\mathrm{NM}=1-3$ & $65.9 \%$ & $51.3-84.6 \%$ & & 2.02 & $1.10-3.71$ & 0.024 & & & \\
\hline Overall number of $\mathrm{NM} \geq 4$ & $44.7 \%$ & $31.7-63.0 \%$ & & 5.90 & $3.68-9.45$ & $<0.001$ & & & \\
\hline \multicolumn{10}{|l|}{ N classification 2} \\
\hline pNO & $83.1 \%$ & $77.6-89.1 \%$ & $<0.001$ & & & & 776.74 & 781.53 & 0.248 \\
\hline Largest diameter of $\mathrm{NM}<20 \mathrm{~mm}$ & $63.2 \%$ & $50.3-79.4 \%$ & & 2.69 & $1.60-4.52$ & $<0.001$ & & & \\
\hline Largest diameter of $\mathrm{NM} \geq 20 \mathrm{~mm}$ & $30.0 \%$ & $14.6-61.6 \%$ & & 8.00 & $4.46-14.32$ & $<0.001$ & & & \\
\hline \multicolumn{10}{|l|}{ N classification 3} \\
\hline pNO & $83.1 \%$ & $77.6-89.1 \%$ & $<0.001$ & & & & 831.96 & 836.89 & 0.291 \\
\hline Low nodal disease burden & $66.2 \%$ & $49.0-89.4 \%$ & & 1.98 & $0.99-3.96$ & 0.05 & & & \\
\hline High nodal disease burden & $44.3 \%$ & $31.8-61.6 \%$ & & 5.83 & $3.67-9.26$ & $<0.001$ & & & \\
\hline \multicolumn{10}{|l|}{ Current N staging (TNM 8th ed.) } \\
\hline pN0 & $82.5 \%$ & $77.1-88.3 \%$ & $<0.001$ & & & & 857.82 & 867.73 & 0.253 \\
\hline pN1 & $53.0 \%$ & $27.7-100 \%$ & & 2.83 & $1.01-7.90$ & 0.047 & & & \\
\hline pN2a & - & - & & 4.48 & $1.38-14.60$ & 0.013 & & & \\
\hline $\mathrm{pN} 2 \mathrm{~b}$ & $53.8 \%$ & $36.6-79.2 \%$ & & 4.21 & $2.29-7.75$ & $<0.001$ & & & \\
\hline pN3 & $50.2 \%$ & $34.1-74.0 \%$ & & 4.89 & $2.91-8.20$ & $<0.001$ & & & \\
\hline
\end{tabular}



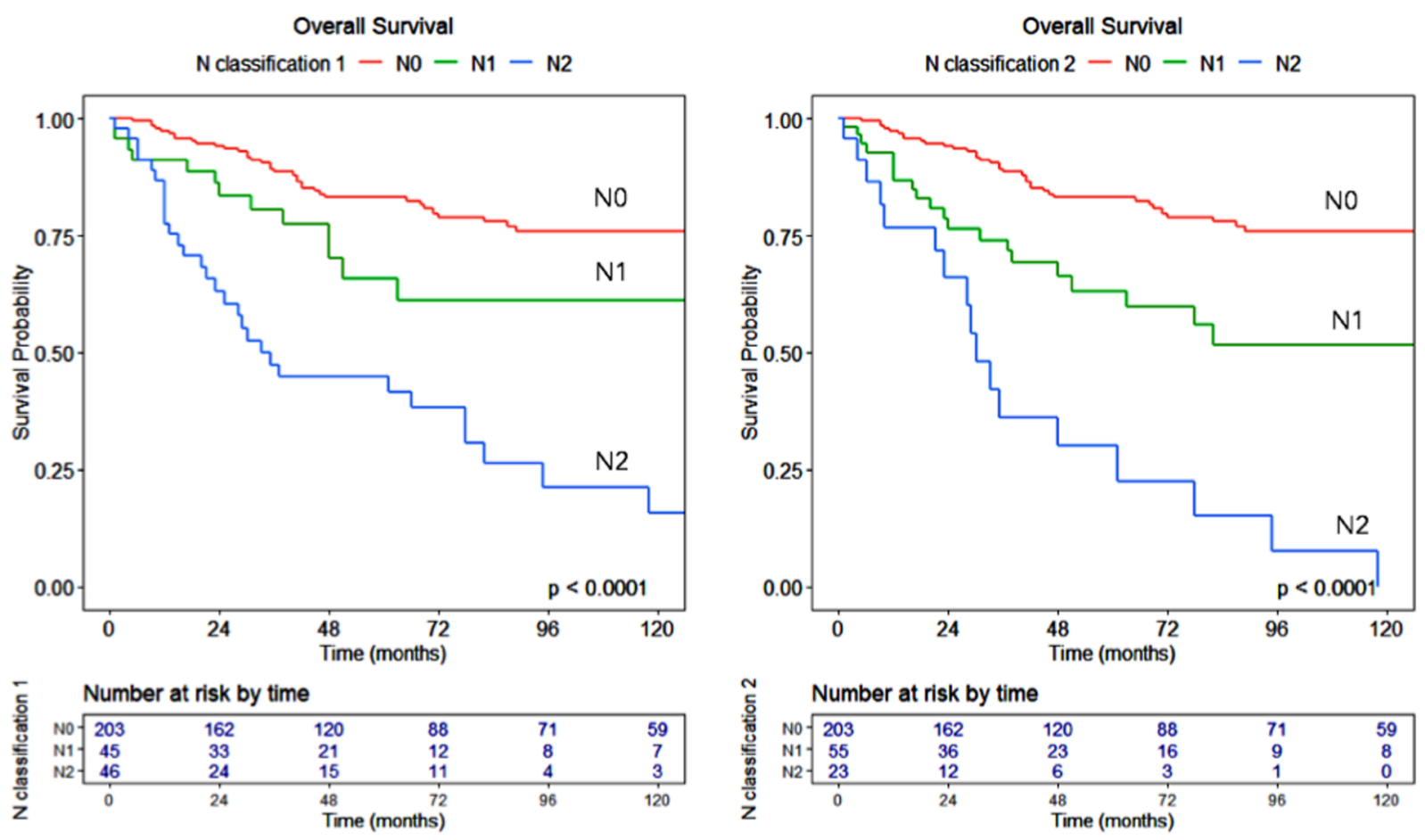

Overall Survival
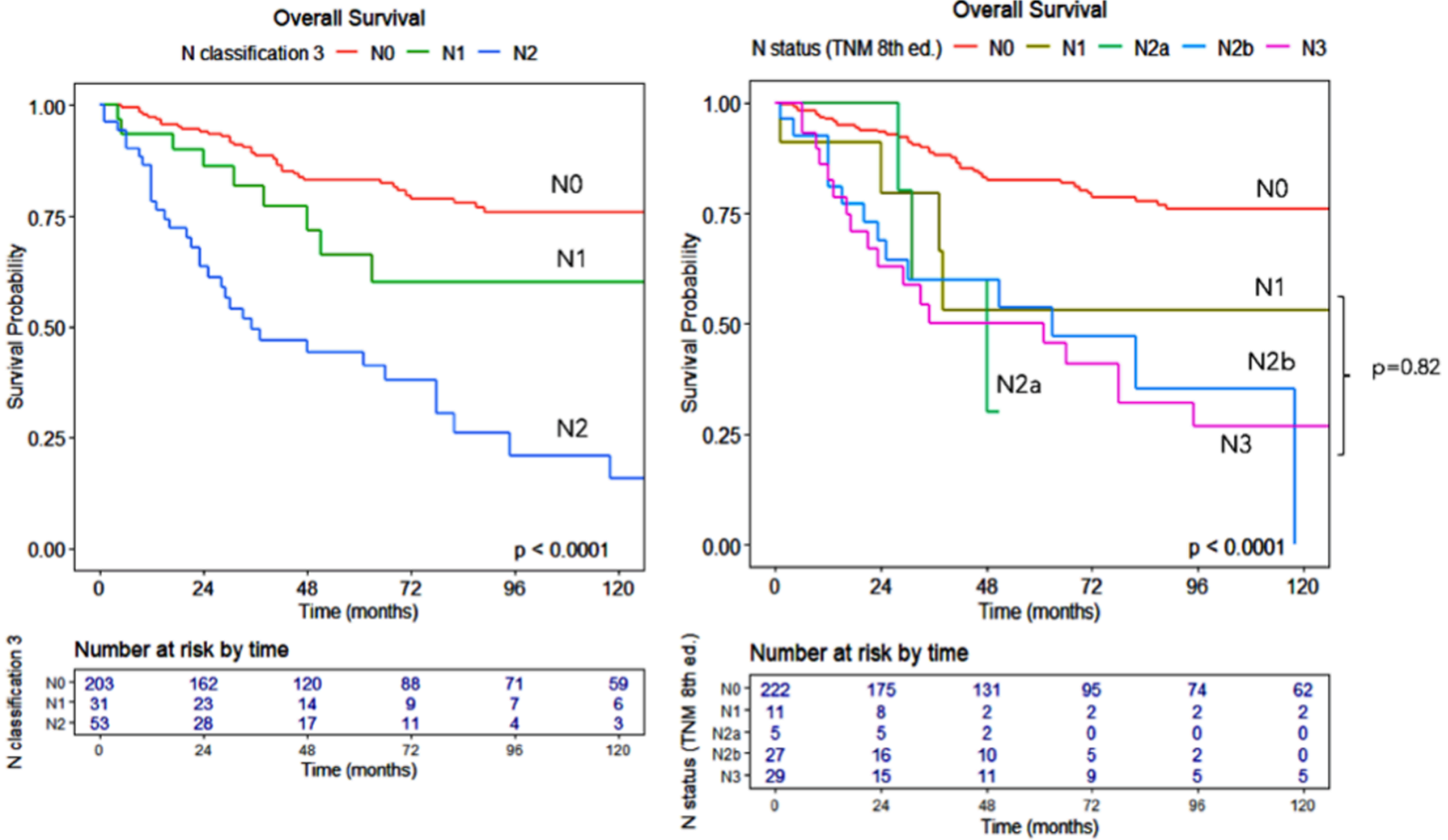

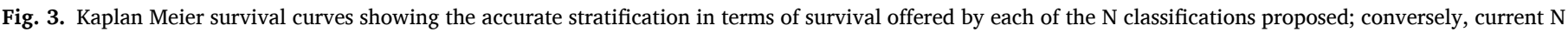

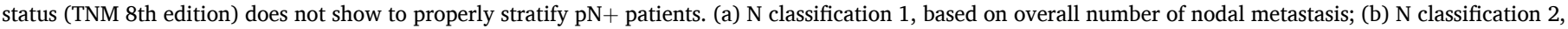

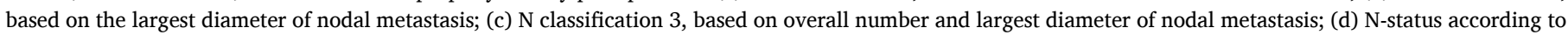
current TNM 8th edition (see also Fig. S4).

confirmed to be strong independent prognostic factors (Table S3).

\section{Discussion}

The present study confirmed that in MSG carcinoma increasing nodal involvement is a strong negative prognostic impact on survival. Data herein presented showed also that current $\mathrm{N}$-categories do not allow for reliable prognostic stratification. This is probably due to the complete absence in clinical reality of some factors defining the levels of the current $\mathrm{N}$-classification (no bilateral nodal involvement nor lymph-node with a diameter $>6 \mathrm{~cm}$ ), and to the lack of prognostic relevance of ENE. Conversely, it highlights the paramount importance of considering 
parotid lymph-nodes as well as lateral cervical lymph-nodes.

\section{Nodal prognostic factors}

Nodal status ( $\mathrm{pN}+\mathrm{vs} \mathrm{pN} 0$ ) is considered among the most important prognostic factors in MSG carcinoma [6,7,27-33]. Our overall rate of nodal involvement was $31.4 \%$, in keeping with previously reported figures, which range between $11.8 \%$ and $37 \%$ [1,4,9,14,30,34]. Interestingly, our series, which also includes patients with exclusive parotid lymph-node involvement, has values comparable with those reported by Klussmann et al. who performed a very similar histologic analysis [9]. In addition to the simple, dichotomic distinction between $\mathrm{NO}$ and $\mathrm{N}+$ patients, we broadened our analysis to identify which nodal factors affected prognosis.

\section{Parotid lymph-nodes}

O'Brien et al. in their pivotal paper on metastatic cutaneous squamous cell carcinoma (SCC) of the parotid gland, should be credited with the first attempt to conceive a specific nodal classification considering also parotid nodes [35]. The issue of metastasis to parotid lymph-nodes when dealing with primary parotid carcinoma is also well known, $[1,9,11,36]$ but the prognostic relevance is probably underestimated. Klussmann et al. discovered that a not negligible rate of patients had parotid lymph-node involvement that proved to increase the risk of recurrence at univariate analysis [9]. Lim et al. clearly demonstrated that: (i) parotid lymph-node involvement was associated with a higher risk of lateral neck involvement, (ii) patients classified as NO but with parotid lymph-node metastasis had a significantly worse DSS and a higher risk of recurrence than patients completely free of any nodal localization [10]. Also, Olsen et al. advocated deep lobe parotidectomy in presence of high-grade parotid cancer and/or in case of superficial parotid lymph-node and/or lateral neck involvement [11].

Our data demonstrate that disease localization to parotid lymphnodes per se implies an increased risk of lateral neck involvement and a significant impact on survival. These findings underline not only the importance of a specific lymphatic drainage pathway in which the parotid gland may be a halfway station between the tumor and the neck, but also the inherent prognostic relevance of parotid nodal involvement. This led us to advance the proposal to include parotid nodes within the new N-classification and, once more, supports the concept that excision of the deep lobe, whenever indicated, should be considered an essential step in the treatment of the primary lesion and of the nodal basin.

\section{Extra-nodal extension}

ENE has been historically considered one of the most important survival predictors in head and neck cancer and for this reason has also been included among nodal prognosticators for salivary gland cancer, despite inconclusive and somewhat conflicting results. Meyer et al. in their analysis on 128 patients with parotid gland cancer, were not able to demonstrate any impact of ENE on survival; moreover, a higher number of ENE+ nodes was not associated with a worse prognosis [16]. Coversely, Yoo et al. found that ENE independently affected the risk of nodal recurrence [34]. Hong et al, in a cohort of 87 patients with high-grade salivary gland cancer, found that ENE independently affected only DFS. [15] Aro et al. [17] and Hsieh et al. [37] demonstrated that ENE did not have any impact on survival. It is worth mentioning, however, that a recent report did identify ENE as one of the most important prognostic predictors [18].

In our cohort of patients, ENE was identified in $61.5 \%$ of cases, higher than the values reported in the literature $(31-57 \%)[5,18,19]$, but it failed to demonstrate any influence on survival. Our data, moreover, seem to suggest that ENE is directly correlated with increasing number and dimension of lymph-nodes (Fig. S2, S3).
Number and dimensions of nodes

In recent years, attention has been directed towards the burden of lymphatic involvement in the field of salivary gland cancer. Consequently, parameters such as number of involved nodes and nodal ratio (NR) were evaluated. Suzuki et al. discovered that a NR of $\geq 0.38$ was associated with a significantly shorter overall survival time [19]. Similarly, Hong et al. showed that a NR $>0.4$ had an independent impact on OS, DFS, and DSS in high-grade salivary gland malignancies [15]. Aro et al., in a National Database analysis on major and minor salivary gland cancers, identified number of positive lymph-nodes as the strongest prognostic factor whereas other nodal factors such as size had no impact on survival [17]. Lee et al. identified number of positive nodes as a main nodal prognostic factor [18].

Our results are in keeping with these recent experiences as regards the number of involved nodes [17,19]. X-tile analysis identified $\geq 4$ lymph-nodes as the cutoff between low- and high nodal burden patients. A similar cutoff was identified by Qian et al., who analyzed 8668 patients with MSG carcinoma from the SEER database, found that $>4$ positive lymph-nodes, and a NR $>0.15$ independently affected DSS [25]. Whilst strongly supporting these findings as regards the number of involved lymph-nodes as a predictor of survival, our experience identifies maximal nodal dimension $\geq 20 \mathrm{~mm}$ rather than ENE as a prognosticator. We decided not to rely upon NR for two reasons: first, the relationship between nodal yield and survival may be not linear, and second, apart from extent of neck dissection, there are other factors that may alter the relationship between nodal yield and NR [38-41].

\section{Proposal of a novel N-classification}

The inability of current $\mathrm{N}$-categories to differentiate between prognostic groups prompted us to redefine and simplify them by including only the number and maximum diameter of metastatic nodes. Recently, two similar attempts have been made: Aro et al. stratified patients in 4 categories mainly according to number of positive nodes: N0, N1 (1-2 $\mathrm{N}+$ ), N2 (3-21 N+), N3 (>22 or ENE + ) [17]. Lee et al., conversely, preferred to stratify their cohort of 172 patients with intermediate/highgrade tumors of major and minor salivary glands into 3 categories: N0, $\mathrm{N} 1(1 \mathrm{LN}+), \mathrm{N} 2(\geq 2 \mathrm{~N}+$ and/or ENE + ) [18]. Both proposals were found to be superior to the current TNM system and allowed a more precise prognostic stratification.

According to our models, we prefer to advance three systems of Nclassification according to the number and/or largest dimension of metastatic lymph-nodes. These models, all of which were found to be better than the current $\mathrm{N}$-categories in prognostic stratification with an independent impact on survival, were conceived to depict the burden of nodal involvement. This was statistically confirmed by all employed indices (AIC, BIC, Nagelkerke pseudo- $\mathrm{R}^{2}$ ) once again demonstrating the superiority of these models compared to the 8th TNM Edition N-classification. Among our 3 models, the second and third are apparently the most promising, but their validation and assessment in an external, larger cohort is essential to identify the most effective one.

\section{Points of strength and limitations of the study}

A point of strength of our analysis is that all histological subtypes and tumor grades, not only intermediate- or high-grade tumors, were included and that only tumors arising in MSG, as dictated by TNM, have been evaluated. Moreover, we did not retrieve data from national databases and, consequently, we were able to analyze in detail all information pertaining to surgical procedure, histologic findings, and pattern of recurrence. Furthermore, we could demonstrate that involvement of parotid lymph-nodes is of paramount prognostic importance.

The main limitation of the study is its retrospective, observational nature. Second, nodal status could be assessed only in those patients who underwent any type of neck dissection. Lastly, the data set in this 
study has been used to define the variables of interest and estimate the prognostic ability of the models constructed. External validation in an independent cohort of patients is required to exclude overfitting and verify the generalizability of our findings.

\section{Conclusions}

In this study, high tumor grade, high T-category and nodal involvement confirmed their prognostic relevance in MSG carcinoma. The current $\mathrm{N}$-classification failed to demonstrate a satisfactory prognostic potential. Conversely, increasing overall number and diameter of nodes, including those contained within the parotid gland, proved to stratify survival of patients more accurately. Three proposals of a novel $\mathrm{N}$-classification based upon number of lymph-nodes, their largest diameter, and a combination of both, have been advanced.

\section{Declaration of Competing Interest}

The authors declare that they have no known competing financial interests or personal relationships that could have appeared to influence the work reported in this paper.

\section{Appendix A. Supplementary material}

Supplementary data to this article can be found online at https://doi. org/10.1016/j.oraloncology.2020.105076.

\section{References}

[1] Armstrong JG, Harrison LB, Thaler HT, et al. The indications for elective treatment of the neck in cancer of the major salivary glands. Cancer 1992;69(3):615-9. https://doi.org/10.1002/1097-0142(19920201)69:3<615::aidcncr2820690303>3.0.c0;2-9.

[2] Medina JE. Neck dissection in the treatment of cancer of major salivary glands. Otolaryngol Clin North Am 1998;31(5):815-22. https://doi.org/10.1016/s00306665(05)70089-x.

[3] Vander Poorten VL, Balm AJ, Hilgers FJ, et al. The development of a prognostic score for patients with parotid carcinoma. Cancer 1999;85(9):2057-67.

[4] Zbären P, Schüpbach J, Nuyens M, Stauffer E, Greiner R, Häusler R. Carcinoma of the parotid gland. Am J Surg 2003;186(1):57-62. https://doi.org/10.1016/s00029610(03)00105-3.

[5] Terhaard CH, Lubsen H, Van der Tweel I, et al. Salivary gland carcinoma: independent prognostic factors for locoregional control, distant metastases, and overall survival: results of the Dutch head and neck oncology cooperative group. Head Neck 2004;26(8):681-93. https://doi.org/10.1002/hed.10400.

[6] Bhattacharyya N, Fried MP. Determinants of survival in parotid gland carcinoma: a population-based study. Am J Otolaryngol 2005;26(1):39-44. https://doi.org/ 10.1016/j.amjoto.2004.06.017.

[7] Honda K, Tanaka S, Shinohara S, et al. Survival in patients with parotid gland carcinoma - results of a multi-center study. Am J Otolaryngol 2018;39(1):65-70. https://doi.org/10.1016/j.amjoto.2017.10.012.

[8] Brierley J, editor. TNM Classification of Malignant Tumours. eighth ed. John Wiley \& Sons, Inc; 2017.

[9] Klussmann JP, Ponert T, Mueller RP, Dienes HP, Guntinas-Lichius O. Patterns of lymph node spread and its influence on outcome in resectable parotid cancer. Eur $\mathrm{J}$ Surg Oncol 2008;34(8):932-7. https://doi.org/10.1016/j.ejso.2008.02.004.

[10] Lim CM, Gilbert MR, Johnson JT, Kim S. Clinical significance of intraparotid lymph node metastasis in primary parotid cancer. Head Neck 2014;36(11):1634-7. https://doi.org/10.1002/hed.23507.

[11] Olsen KD, Moore EJ. Deep lobe parotidectomy: clinical rationale in the management of primary and metastatic cancer. Eur Arch Otorhinolaryngol 2014; 271(5):1181-5. https://doi.org/10.1007/s00405-013-2616-8.

[12] Kelley DJ, Spiro RH. Management of the neck in parotid carcinoma. Am J Surg 1996;172(6):695-7. https://doi.org/10.1016/s0002-9610(96)00307-8.

[13] Westergaard-Nielsen M, Rosenberg T, Gerke O, Dyrvig AK, Godballe C, Bjørndal K. Elective neck dissection in patients with salivary gland carcinoma: a systematic review and meta-analysis [published online ahead of print, 2020 May 20]. J Oral Pathol Med 2020. https://doi.org/10.1111/jop.13034.

[14] Ali S, Palmer FL, DiLorenzo M, Shah JP, Patel SG, Ganly I. Treatment of the neck in carcinoma of the parotid gland. Ann Surg Oncol 2014;21(9):3042-8. https://doi. org/10.1245/s10434-014-3681-y.

[15] Hong HR, Roh JL, Cho KJ, Choi SH, Nam SY, Kim SY. Prognostic value of lymph node density in high-grade salivary gland cancers. J Surg Oncol 2015;111(6): 784-9. https://doi.org/10.1002/jso.23874.

[16] Meyer MF, Kreppel M, Meinrath J, et al. Prediction of outcome by lymph node ratio in patients with parotid gland cancer. Clin Otolaryngol 2017;42(1):98-103. https://doi.org/10.1111/coa.12672.
[17] Aro K, Ho AS, Luu M, et al. Development of a novel salivary gland cancer lymph node staging system. Cancer 2018;124(15):3171-80. https://doi.org/10.1002/ cncr.31535.

[18] Lee H, Roh JL, Cho KJ, Choi SH, Nam SY, Kim SY. Positive lymph node number and extranodal extension for predicting recurrence and survival in patients with salivary gland cancer. Head Neck. 2020;42(8):1994-2001. https://doi.org/ 10.1002/hed.26135.

[19] Suzuki H, Hanai N, Hirakawa H, Nishikawa D, Hasegawa Y. Lymph node density is a prognostic factor in patients with major salivary gland carcinoma. Oncol Lett. 2015;10(6):3523-8. https://doi.org/10.3892/ol.2015.3814.

[20] Brandwein MS, Ivanov K, Wallace DI, et al. Mucoepidermoid carcinoma: a clinicopathologic study of 80 patients with special reference to histological grading. Am J Surg Pathol 2001;25(7):835-45. https://doi.org/10.1097/ grading. Am J Surg Pathol 2001 ;

[21] Perzin KH, Gullane P, Clairmont AC. Adenoid cystic carcinomas arising in salivary glands: a correlation of histologic features and clinical course. Cancer 1978;42(1): 265-82. https://doi.org/10.1002/1097-0142(197807)42:1<265::aidcncr2820420141>3.0.co;2-z.

[22] Szanto PA, Luna MA, Tortoledo ME, White RA. Histologic grading of adenoid cystic carcinoma of the salivary glands. Cancer 1984;54(6):1062-9. https://doi.org/ 10.1002/1097-0142(19840915)54:6<1062::aid-cncr2820540622>3.0.co;2-e.

[23] El-Naggar AK, Chan JK, Grandis JR, et al. WHO classification of head and neck tumours. IARC Who Classification of Tum 2017.

[24] Lombardi D, McGurk M, Vander Poorten V, et al. Surgical treatment of salivary malignant tumors. Oral Oncol 2017;65:102-13. https://doi.org/10.1016/j. oraloncology.2016.12.007.

[25] Qian K, Sun W, Guo K, et al. The number and ratio of positive lymph nodes are independent prognostic factors for patients with major salivary gland cancer: results from the surveillance, epidemiology, and End Results dataset. Eur J Surg Oncol 2019;45(6):1025-32. https://doi.org/10.1016/j.ejso.2018.11.008.

[27] Poulsen MG, Pratt GR, Kynaston B, Tripcony LB. Prognostic variables in malignant epithelial tumors of the parotid. Int J Radiat Oncol Biol Phys 1992;23(2):327-32. https://doi.org/10.1016/0360-3016(92)90749-8.

[28] Chakrabarti S, Nair D, Malik A, et al. Prognostic factors in parotid cancers: clinicopathological and treatment factors influencing outcomes. Indian $\mathrm{J}$ Cancer 2018;55(1):98-104. https://doi.org/10.4103/ijc.IJC_503_17.

[29] Bhattacharyya N, Fried MP. Nodal metastasis in major salivary gland cancer: predictive factors and effects on survival. Arch Otolaryngol Head Neck Surg 2002; 128(8):904-8. https://doi.org/10.1001/archotol.128.8.904.

[30] Stenner M, Molls C, Luers JC, Beutner D, Klussmann JP, Huettenbrink KB. Occurrence of lymph node metastasis in early-stage parotid gland cancer. Eur Arch Otorhinolaryngol 2012;269(2):643-8. https://doi.org/10.1007/s00405-011-16632.

[31] Chang JW, Hong HJ, Ban MJ, et al. Prognostic factors and treatment outcomes of parotid gland cancer: a 10-year single-center experience. Otolaryngol Head Neck Surg 2015;153(6):981-9. https://doi.org/10.1177/0194599815594789.

[32] Lombardi D, Accorona R, Lambert A, et al. Long-term outcomes and prognosis in submandibular gland malignant tumors: a multicenter study. Laryngoscope 2018; 128(12):2745-50. https://doi.org/10.1002/lary.27236.

[33] Paderno A, Tomasoni M, Mattavelli D, Battocchio S, Lombardi D, Nicolai P. Primary parotid carcinoma: analysis of risk factors and validation of a prognostic index. Eur Arch Otorhinolaryngol 2018;275(11):2829-41. https://doi.org/ 10.1007/s00405-018-5122-1.

[34] Yoo SH, Roh JL, Kim SO, et al. Patterns and treatment of neck metastases in patients with salivary gland cancers. J Surg Oncol 2015;111(8):1000-6. https:// doi.org/10.1002/jso.23914.

[35] O'Brien CJ, McNeil EB, McMahon JD, Pathak I, Lauer CS, Jackson MA. Significance of clinical stage, extent of surgery, and pathologic findings in metastatic cutaneous squamous carcinoma of the parotid gland. Head Neck 2002;24(5):417-22. https:// doi.org/10.1002/hed.10063.

[36] Stenner M, Molls C, Klussmann JP, Hüttenbrink KB. Zur Prognose chirurgisch therapierter primärer Parotiskarzinome - eine Untersuchung an 231 Fällen [Prognosis of surgically treated primary parotid gland cancer - an evaluation of 231 cases]. Laryngorhinootologie 2011;90(11):664-71. https://doi.org/10.1055/s0031-1285924.

[37] Hsieh CE, Hung CY, Lin CY, et al. High metastatic node number, not extranodal extension, as a node-related prognosticator in surgically treated patients with nodal metastatic salivary gland carcinoma. Head Neck. 2019;41(6):1572-82. https://doi.org/10.1002/hed.25603.

[38] Ebrahimi A, Zhang WJ, Gao K, Clark JR. Nodal yield and survival in oral squamous cancer: defining the standard of care. Cancer 2011;117(13):2917-25. https://doi. org/10.1002/cncr.25834.

[39] Ebrahimi A, Clark JR, Amit M, et al. Minimum nodal yield in oral squamous cell carcinoma: defining the standard of care in a multicenter international pooled validation study. Ann Surg Oncol 2014;21(9):3049-55. https://doi.org/10.1245/ s10434-014-3702-x.

[40] Marres CC, de Ridder M, Hegger I, et al. The influence of nodal yield in neck dissections on lymph node ratio in head and neck cancer. Oral Oncol 2014;50(1): 59-64. https://doi.org/10.1016/j.oraloncology.2013.09.014.

[41] Roberts TJ, Colevas AD, Hara W, Holsinger FC, Oakley-Girvan I, Divi V. Number of positive nodes is superior to the lymph node ratio and American joint committee on Cancer $\mathrm{N}$ staging for the prognosis of surgically treated head and neck squamous cell carcinomas. Cancer 2016;122(9):1388-97. https://doi.org/ 10.1002/cncr.29932. 